\title{
Prediction of Outcomes in Mini-Basketball Training Program for Preschool Children with Autism Using Machine Learning Models
}

\author{
Zhiyuan Sun ${ }^{1,2}$, Fabian Herold ${ }^{3,4}$, Kelong Cai ${ }^{1,2}$, Qian Yu ${ }^{5}$, Xiaoxiao Dong ${ }^{1,2}$, Zhimei Liu ${ }^{1,2}$, \\ Jinming $\mathrm{Li}^{6}$, Aiguo Chen ${ }^{1,2, *}$ and Liye $\mathrm{Zou}^{7, *}$ \\ ${ }^{1}$ College of Physical Education, Yangzhou University, Yangzhou, 225127, China \\ ${ }^{2}$ Institute of Sports, Exercise and Brain, Yangzhou University, Yangzhou, 225127, China \\ ${ }^{3}$ Department of Neurology, Medical Faculty, Otto von Guericke University, Magdeburg, 39120, Germany \\ ${ }^{4}$ German Center for Neurodegenerative Diseases (DZNE), Research Group Neuroprotection, Magdeburg, 39120, Germany \\ ${ }^{5}$ Faculty of Education, University of Macau, Macao, China \\ ${ }^{6}$ Exercise Psychophysiology Laboratory, Institute of KEEP Collaborative Innovation, School of Psychology, Shenzhen University, \\ Shenzhen, 518060, China \\ ${ }^{7}$ School of Physical Education and Health, East China Normal University, Shanghai, 200241, China \\ *Corresponding Authors: Aiguo Chen. Email: agchen@yzu.edu.cn; Liye Zou. Email: liyezou123@gmail.com
}

Received: 30 October 2021 Accepted: 24 November 2021

\begin{abstract}
In recent years evidence has emerged suggesting that Mini-basketball training program (MBTP) can be an effective intervention method to improve social communication (SC) impairments and restricted and repetitive behaviors (RRBs) in preschool children suffering from autism spectrum disorder (ASD). However, there is a considerable degree if interindividual variability concerning these social outcomes and thus not all preschool children with ASD profit from a MBTP intervention to the same extent. In order to make more accurate predictions which preschool children with ASD can benefit from an MBTP intervention or which preschool children with ASD need additional interventions to achieve behavioral improvements, further research is required. This study aimed to investigate which individual factors of preschool children with ASD can predict MBTP intervention outcomes concerning SC impairments and RRBs. Then, test the performance of machine learning models in predicting intervention outcomes based on these factors. Participants were 26 preschool children with ASD who enrolled in a quasi-experiment and received MBTP intervention. Baseline demographic variables (e.g., age, body, mass index [BMI]), indicators of physical fitness (e.g., handgrip strength, balance performance), performance in executive function, severity of ASD symptoms, level of SC impairments, and severity of RRBs were obtained to predict treatment outcomes after MBTP intervention. Machine learning models were established based on support vector machine algorithm were implemented. For comparison, we also employed multiple linear regression models in statistics. Our findings suggest that in preschool children with ASD symptomatic severity $(r=0.712, p<$ $0.001)$ and baseline SC impairments $(r=0.713, p<0.001)$ are predictors for intervention outcomes of SC impairments. Furthermore, BMI $(r=-0.430, p=0.028)$, symptomatic severity $(r=0.656, p<0.001)$, baseline SC impairments $(r=0.504, p=0.009)$ and baseline RRBs $(r=0.647, p<0.001)$ can predict intervention outcomes of RRBs. Statistical models predicted $59.6 \%$ of variance in post-treatment SC impairments (MSE $=0.455, \mathrm{RMSE}=0.675$, $\left.\mathrm{R}^{2}=0.596\right)$ and $58.9 \%$ of variance in post-treatment $\mathrm{RRBs}\left(\mathrm{MSE}=0.464, \mathrm{RMSE}=0.681, \mathrm{R}^{2}=0.589\right)$. Machine learning models predicted $83 \%$ of variance in post-treatment SC impairments $(\mathrm{MSE}=0.188, \mathrm{RMSE}=0.434$, $\mathrm{R}^{2}=0.83$ ) and $85.9 \%$ of variance in post-treatment $\mathrm{RRBs}\left(\mathrm{MSE}=0.051\right.$, $\left.\mathrm{RMSE}=0.226, \mathrm{R}^{2}=0.859\right)$, which were better than statistical models. Our findings suggest that baseline characteristics such as symptomatic severity of
\end{abstract}


ASD symptoms and SC impairments are important predictors determining MBTP intervention-induced improvements concerning SC impairments and RBBs. Furthermore, the current study revealed that machine learning models can successfully be applied to predict the MBTP intervention-related outcomes in preschool children with ASD, and performed better than statistical models. Our findings can help to inform which preschool children with ASD are most likely to benefit from an MBTP intervention, and they might provide a reference for the development of personalized intervention programs for preschool children with ASD.

\section{KEYWORDS}

Prediction; outcomes; mini-basketball training program; autistic children; machine learning models

\section{Introduction}

Autism spectrum disorder (ASD) is a life-long neurodevelopmental disability that develops during early childhood [1]. There are two core symptoms of ASD namely (i) social communication impairments, and (ii) restricted and repetitive behaviors. Social communication (SC) impairments are typically manifested by the inability to communicate and interact with people through facial expressions and other social skills including difficulties in establishing a normal social relationship with family members and in developing friendships [2]. Restricted and repetitive behaviors (RBBs) in individuals suffering from ASD refer to repetitive and monotonous behaviors of craving for the environment at a high frequency and in a fixed way [3], which, in turn, negatively affects the acquisition of functional behaviors and other social skills, thereby causing negative health effects such as self-harm emotions [4,5]. Based on data from the Centers for Disease Control and Prevention in 2020, the prevalence of ASD in children has increased to 1/54 [6], and a metaanalysis in 2018 found that the prevalence of ASD in Chinese children was 2.6\% [7]. Thus, this is a major public health problem that seriously influence survival outcomes and the development of a children.

Physical exercise is an effective intervention strategy to treat ASD in preschool children [8,9]. In this context, game-based physical exercises such as basketball, moved in the focus of ASD researcher since this type of physical exercise has several advantages (e.g., easy to learn, facilitate social skills due to the team play). Indeed, Mini-basketball training program (MBTP) has been shown to improve SC impairments and RRBs in preschool children with ASD and positively influence the brain (e.g., white matter) [10-12]. It is worth noting that there is a considerable degree of interindividual variability in response to physical interventions. For instance, a study investigating the effect yoga in ASD children aged 5 to 12 years, reported that not all participants benefitted equally from the intervention [13]. In particular, $19 \%$ of the participants did not satisfactorily benefit from the intervention in terms of relaxation response-based yoga [13]. Therefore, it seems reasonable to hypothesize that in preschool children with ASD a considerable amount of interindividual variability concerning intervention-induced changes in specific outcomes can be observed (e.g., in response to MBTP concerning SC impairments and RBBs). In order to better individualized physical interventions (such as MBTP) in preschool children with ASD, predicting the outcomes after the intervention is required.

In this regard, two basic questions should be kept in mind: (i) What factors should be used to predict the intervention outcomes? and (ii) What methods should be used to predict the intervention outcomes?

Currently, only a few studies have evaluated the efficacy of MBTP intervention in preschool children with ASD to influence SC impairments and RRBs and observed positive effects of MBTP on these outcome parameters [10-12]. However, factors that can predict the intervention outcomes of MBTP and thus the success of this intervention method has, so far, not been extensively studied. Although there is 
currently a paucity of research in this direction, other studies in ASD children have yielded some meaningful insights. Concerning demographic information, Bishop et al. [14] found that stereotyped behaviors exist in children over 5 years of age with non-verbal IQ below 70, however, the frequency of stereotyped behaviors decreased with age in normally developing children. Hiller et al. [15] reported that compared with boys, girls have the ability to integrate non-verbal and verbal acts, maintain their ability to talk to each other, and show less and less typical narrow interests. Furthermore, ASD children were more likely to be overweight and obese than normal children [16]. Regarding executive functions, ASD children are significantly different from those of normal children, and it has been observed that deficits in executive functioning contribute, at least partly, to SC impairments and RRBs [17]. In addition, the higher the severity of symptoms for ASD children, the more severe the core symptoms tend to be, and their physical health levels being significantly lower than those of normal children [18,19]. Collectively, the above-mentioned evidence suggests that in preschool children with ASD, demographic variables, performance of executive functioning, physical health and symptomatic severity might influence the response to a physical intervention (i.e., MBTP) and thus being important predictor variables that need to be considered.

With increasing data availability and complexity, the predictive performance of traditional statistical methods is limited. Therefore, machine learning methods have been proposed. Machine learning, a branch of artificial intelligence, enables computers to learn from data and have better fitting ability to the data [20]. Machine learning has been widely used in various fields, for example, to prevent sports injuries [21] and to predict stock prices [22]. Moreover, machine learning has shown to perform well in predicting psychiatric intervention outcomes $[23,24]$, and has already contribute to achievements in areas such as depression and obsessive-compulsive disorder research [25,26]. Compared to traditional statistical methods, although machine learning is difficult to make causal inferences, it is superior when it comes to predict complex data [27], as its "algorithm" does not rely on data assumptions of linear, normal distribution and homogeneity of variance [28]. Usually, machine learning models divide the original data set into two subsets, training and test sets, based on a certain proportion, establishes a prediction model in the training set, and performs multiple data fitting and optimization tests, to determine if the model best reflects data characteristics. Finally, samples from the test set are input into the model obtained from the training set to evaluate and verify the performance of the model. In short, machine learning methods maybe helpful in predicting intervention outcomes (e.g., of MBTP in preschool children with ASD), and are perhaps more accurate and reliable than conventional statistical methods.

As mentioned, not all preschool children with ASD benefit equally from physical interventions (e.g., MBTP) and in order to maximize the intervention related benefits it is of great importance to better predict the intervention response concerning specific outcome parameters (e.g., SC impairments and RBBs in preschool children with ASD). Such a deeper understanding of prediction of intervention outcomes, in turn, allow to better personalize the interventions. Thus, this study aims to investigate which factors in preschool children with ASD are associated with the treatment outcomes in SC impairments and RBBs in response to a MBTP intervention. And then through these factors, statistical models and machine learning models were implemented to predict the intervention outcomes in SC impairments and RRBs in preschool children with ASD in response to a MBTP. Furthermore, we compared the performance of the two models. Presumably, our findings will aid the identification of preschool children with ASD that are most likely to benefit from MBTP and provide reference for the development of personalized intervention programs for preschool children with ASD.

\section{Methods}

\subsection{Participants}

Ninety-four preschool children with DSM-5-diagnosed ASD from two education centers (Yangzhou, China) participated in a quasi-experiment [10]. Children were eligible if they met all of the following 
inclusion criteria: (i) Han nationality, (ii) age 3-6 years old, (iii) all participants need the consent of their guardians to participate in this experiment. Afterwards, these individuals were further screened and excluded if they: (i) received a basketball training or regular physical training in the past 6 months, (ii) have additional psychiatric and/or neurological diseases (e.g., epilepsy, phenylketonuria), (iii) suffer from visual and hearing impairments, (iv) have had a history of head trauma, or (v) suffer from health condition that does them not allow to perform physical exercise (e.g., heart insufficiency). According to the above-mentioned exclusion criteria, a total of 59 participants were included in the study and geographically assigned to the experimental $(n=30)$ or control $(n=29)$ group. As some participants failed to complete behavioral tests in later study phases, completed data sets of 41 participants (experimental group $(\mathrm{n}=26)$ and control group $(\mathrm{n}=15)$ (see Fig. A1)) were available. In the current study, the data of the 26 preschool children with ASD who were allocated to the experimental group receiving the MBTP intervention were used for further data analysis, and the data of the 15 preschool children with ASD in the control group were excluded because these children did not receive the MBTP intervention.

\subsection{Mini-Basketball Training Program}

The MBTP intervention was conducted by two certified physical education educators and more details of the operation could be found in already published articles [10,11]. In brief, the MBTP intervention can be divided into three phases: (i) improving interest and standardizing classroom routines, (ii) improving MBTP skills and social communication abilities, and (iii) improving cooperative abilities and, social skills to achieve a better integration in social groups (see Fig. 1). A single exercise session was structured as follows: (i) an introduction part, (ii) part in which warm-up exercise was performed, (iii) a part in which MBTP was conducted, and (iv) a relaxation part. The MBTP was performed 5 times a week for a total of 12 weeks. Each sessions lasted $40 \mathrm{~min}$, and the exercise intensity can be rated as moderate, which average heart rate is controlled at $128 \sim 148$ beats $/ \mathrm{min}$.

\begin{tabular}{|c|c|c|c|}
\hline Phase & Goal & Content & Duration \\
\hline Phase I & $\begin{array}{c}\text { Standardize classroom } \\
\text { routines } \\
\text { Increase children's interest in } \\
\text { mini- basketball }\end{array}$ & $\begin{array}{c}\text { Classroom routines (taking turns, waiting, } \\
\text { obeying, etc.) } \\
\text { Simple basketball training (roll and throw } \\
\text { the ball, etc.) }\end{array}$ & 2 weeks \\
\hline Phase II & $\begin{array}{l}\text { Improve children's } \\
\text { mini-basketball skills } \\
\text { Improve their social } \\
\text { communication skills }\end{array}$ & $\begin{array}{l}\text { Basic basketball skill (dribbling, passing, } \\
\text { shooting, etc.) } \\
\text { Peer coordination training (passing and } \\
\text { catching ball, relay racing, etc.) }\end{array}$ & 8 weeks \\
\hline Phase III & $\begin{array}{l}\text { Improve children's } \\
\text { cooperative ability, social } \\
\text { skills, and collectivization }\end{array}$ & $\begin{array}{l}\text { Group game based on mini-basketball } \\
\text { (basketball-dribbling relay, } \\
\text { basketball-passing relays, basket-moving } \\
\text { shooting, playing ducks, etc.) }\end{array}$ & 2 weeks \\
\hline
\end{tabular}

Figure 1: Mini-basketball training program [11]

\subsection{Measures}

Baseline demographic information, including sex, age and body mass index (BMI) for preschool children with ASD were obtained. The Childhood Autism Rating Scale (CARS) [29] and clinical assessment reports were used to assess symptomatic severity. The total score of the scale was 60 , and the scoring criteria were: no autism (total score $<30$ ), mild to moderate autism $(30-37)$, severe autism (37-60, and at least 5 items had a score above 3).

The National Standard Manual of Physical Fitness Measurement (Early Childhood Part) was used to evaluate physical health development of preschool children with ASD [12]. Tests included dexterity, 
muscle strength, flexibility and balance. Dexterity test involved $10 \mathrm{~m}$ shuttle run (s), muscle strength test involved standing long jump $(\mathrm{cm})$, flexibility test involved sit and reach $(\mathrm{cm})$, while balance test involved balance beam test (s).

The Childhood Executive Functioning Inventory (CHEXI) [30] was used to assess executive functions in preschool children with ASD. This scale has good reliability and validity and can be used as an assessment tool for Chinese children's executive function [31]. There were 24 items on the scale, and each item had a score of 1 to 5 . It was divided into four sub-scales of working memory planning ability, regulation ability and inhibition ability.

The Social Responsiveness Scale Second Edition (SRS-2) [32] was used to assess SC impairments of preschool children with ASD. This scale is reliable and valid [32]. The 65-item scale has 5 sub-scales (social awareness, social cognition, social communication, social motivation, restrictive interests and repetitive behaviors). The scale calculated the total score and the higher the score, the serious social ability impairments was.

The Repetitive Behavior Scale-Revised (RBS-R) [33] was used to assess RRBs of preschool children with ASD. This scale has good internal consistency and test-retest reliability [34] and was completed by parents/guardians of preschool children with ASD. The scale consisted of 43 items and was divided into 6 sub-dimensions (stereotypic behavior, self-injury, compulsive behavior, ritual behavior, monotonous behavior, and restrictive behavior). Total score was recorded and the higher the score, the serious the repeated and stereotyped behavior.

\subsection{Predictors}

At baseline, demographic information (i.e., sex, age, BMI), symptomatic severity, SC impairments, RRBs, physical fitness (i.e., dexterity, muscle strength, flexibility and balance), and executive functions (i.e., working memory, planning ability, regulation ability and inhibition ability) of preschool children with ASD were assessed. These factors were taken as candidate factors, and normalized via the Z-score standardization method [35] to solve the problem of dimensionless difference between variables.

Python 3.7.4 was used for the further analysis. The predictor is extracted by the Pearson correlation coefficient and $T$ test in the filtering method [36]. Candidate factors with $p<0.05$ were selected as factors which can predict MBTP intervention-related outcomes in SC impairments and RRBs in our cohort of preschool children with ASD.

\subsection{Intervention Outcomes}

The Social Responsiveness Scale Second Edition (SRS-2) and Repetitive Behavior Scale-Revised (RBS-R) were used to evaluate SC impairments and RRBs for preschool children with ASD after receiving MBTP intervention, and scale score (total score) was used to reflect intervention outcomes of MBTP on SC impairments and RRBs of preschool children with ASD.

\subsection{Data Analysis}

In this study, we used two different methods to predict the MBTP-related intervention outcomes in SC impairment and RRBs. As first method, multiple linear regression models were calculated using SPSS 23.0. As second method, machine learning models were determined using Python 3.7.4 (see Sections 2.6.1 and 2.6.2 for more details).

\subsubsection{Algorithm Selection for Machine Learning Models}

In this study, we used support vector machine (SVM) algorithm given that they performed well in the prediction of psychiatric treatment outcomes [23]. SVM algorithm used the principle of structural risk minimization, which could still have good fitting and generalization abilities under small samples [37]. 
The final prediction result of the SVM model was determined by a few support vectors and has a good robustness as it is relatively insensitive to outliers [38]. Moreover, SVM obtained the global minimum of the objective function through reliable known algorithms and expressed the learning problem as a convex optimization problem.

\subsubsection{Establishment Process of the Machine Learning Models}

The Sklearn package in Python 3.7.4 was used to implement machine learning models to predict the MBTP intervention-related outcomes in SC impairment and RRBs.

In this context, the following specific modeling processes were used:

(1) Division of sample sets into training and test set

The train test split method in Sklearn packages was used to randomly divide all sample sets into training set and test set, where the training set was $75 \%$ of the total sample $(n=19)$ and the test set was $25 \%$ of the total sample $(\mathrm{n}=7)$.

(2) Based on the training set, SVM models were established and evaluation indices selected

The Gaussian radial basis function [39] was selected as the kernel function to establish SVM models.

Mean square error (MSE), root mean square error (RMSE) and coefficient of determination $\left(\mathrm{R}^{2}\right)$ [40-42] were used to evaluate the performance of the models.

MSE was expressed as:

$M S E=\frac{1}{m} \sum_{i=1}^{m}\left(y-y^{\prime}\right)^{2}$

RMSE was expressed as:

$R M S E=\sqrt{\frac{1}{m} \sum_{i=1}^{m}\left(y-y^{\prime}\right)^{2}}$

where $\mathrm{m}$ is the total number of samples, $\mathrm{y}$ is the actual value, and $\mathrm{y}^{\prime}$ is the predicted value. The closer MSE and RMSE are to 0, the higher the models' performance.

$\mathrm{R}^{2}$ was expressed as:

$R^{2}=1-\frac{\sum\left(y-y^{\prime}\right)^{2}}{\sum(y-\bar{y})^{2}}$

where $y$ is the actual value, $y^{\prime}$ is the predicted value, and $\bar{y}$ is the average value of the actual value. The closer $\mathrm{R}^{2}$ is to 1 , the higher the models' performance.

(3) Grid search and cross validation

Grid search was used to generate a list of all possible values of each parameter in the estimation function, after which the values in each list were combined to generate a grid. Each grid was used as a training model, and performance was evaluated by 10 -fold cross validation method to optimize the learning algorithm. After the fitting function had tried all combination results, it returned the most suitable learner and automatically adjusted to the best parameter combination.

(4) Inputting the test set to obtain the prediction results of the models, after which the performance of the models was evaluated 


\section{Results}

\subsection{Predictors of Intervention-Related Outcomes for Two Core Symptoms in Preschool Children with ASD}

\subsubsection{Predictors of Intervention-Related Outcomes for SC Impairments in Preschool Children with ASD}

With respect to demographic information, we did not observe significant sex-related difference concerning SC impairments $\left(t_{(24)}=-0.796, p>0.05\right)$. Moreover, Pearson's product difference correlation coefficient between age, BMI and SC impairments did not reach statistical significance. Regarding physical fitness and executive functions, we did not identify candidate factors given that these variables are not significantly correlated with SC impairments in this study. Symptomatic severity $(r=0.712$, $p<0.001)$ and baseline SC impairments $(r=0.713, p<0.001)$ are positively correlated with treatment outcomes for SC impairments (see Table 1). Based on the above-mentioned results, symptomatic severity and baseline SC impairments were selected as predictors concerning treatment outcomes of SC impairments in response to MBTP intervention in preschool children with ASD.

Table 1: Correlation between candidate factors and intervention-related outcomes of SC impairments

\begin{tabular}{lll}
\hline Candidate factors & $r$ & $p$ \\
\hline Age & 0.107 & 0.323 \\
Body Mass Index & -0.371 & 0.062 \\
Dexterity & -0.248 & 0.222 \\
Muscle strength & -0.123 & 0.550 \\
Flexibility & -0.114 & 0.580 \\
Balance & -0.067 & 0.744 \\
Working memory & 0.200 & 0.328 \\
Planning ability & 0.380 & 0.056 \\
Regulation ability & 0.387 & 0.051 \\
Inhibition ability & -0.073 & 0.721 \\
Severity of symptoms & 0.712 & $<0.001$ \\
Baseline SC impairment & 0.713 & $<0.001$ \\
Baseline RRBs & 0.301 & 0.135 \\
\hline Note: $p<0.05$ was considered significant. & &
\end{tabular}

\subsubsection{Predictors of Intervention-Related Outcomes for RRBs in Preschool Children with ASD}

We did not observe significant difference in SC impairments between female and male participants $\left(t_{(24)}=-1.416, p>0.05\right)$. With regard to the correlation analysis, we noticed that there is no significant correlation between age and RRBs, but between BMI and RRBs $(r=-0.430, p=0.028)$. Regarding physical fitness and executive functions, there were no candidate factors that were found to be significantly correlated with treatment outcomes of RRBs. In addition, symptomatic severity $(r=0.656$, $p<0.001)$, baseline SC impairments $(r=0.504, p=0.009)$, and baseline RRBs $(r=0.647, p<0.001)$ has been observed to be positively correlated with treatment outcomes for RRBs (see Table 2). Based on the above-presented results, BMI, symptomatic severity, baseline SC impairments, and baseline RRBs were selected as predictors concerning outcomes of RRBs in response to MBTP in preschool children with ASD. 
Table 2: Correlations between candidate factors and intervention-related outcomes of RRBs

\begin{tabular}{lll}
\hline Candidate factors & $r$ & $p$ \\
\hline Age & 0.238 & 0.242 \\
Body mass index & -0.430 & 0.028 \\
Dexterity & -0.282 & 0.164 \\
Muscle strength & 0.055 & 0.791 \\
Flexibility & 0.308 & 0.126 \\
Balance & -0.085 & 0.681 \\
Working memory & 0.247 & 0.224 \\
Planning ability & 0.211 & 0.300 \\
Regulation ability & 0.302 & 0.133 \\
Inhibition ability & 0.181 & 0.377 \\
Severity of symptoms & 0.656 & $<0.001$ \\
Baseline SC impairment & 0.504 & 0.009 \\
Baseline RRBs & 0.647 & $<0.001$ \\
\hline Note: $p<0.05$ was considered significant. & &
\end{tabular}

\subsection{Prediction Results of Statistical Models Concerning Intervention-Related Outcomes of Two Core Symptoms in Preschool Children with ASD}

Symptomatic severity and baseline SC impairments were included in the multiple linear step-by-step regression model of treatment outcomes for SC impairments. Symptomatic severity $(\beta=0.416$, $p=0.035)$ and baseline SC impairments $(\beta=0.422, p=0.033)$ can predicted the treatment outcomes for $\mathrm{SC}$ impairments. The model (MSE $\left.=0.455, \mathrm{RMSE}=0.675, \mathrm{R}^{2}=0.596\right)$ predicted $59.6 \%$ of the variance in post-treatment SC impairments.

Symptomatic severity, BMI, baseline SC impairments, and baseline RRBs were included in the multiple linear step-by-step regression model of treatment outcomes for RRBs. Baseline SC impairments $(p=0.408)$, BMI $(p=0.112)$ were no statistically significant predictors, while symptomatic severity $(\beta=0.461$, $p=0.005)$ and baseline RRBs $(\beta=0.444, p=0.007)$ can predicted the treatment outcomes for RRBs. The model $\left(\mathrm{MSE}=0.464\right.$, RMSE $\left.=0.681, \mathrm{R}^{2}=0.589\right)$ predicted $58.9 \%$ of the variance in post-treatment RRBs.

\subsection{Prediction Results of Machine Learning Models Concerning Intervention-Related Outcomes of Two Core Symptoms in Preschool Children with ASD}

\subsubsection{Prediction Results of Machine Learning Models Concerning Intervention-Related Outcomes of SC Impairments in Preschool Children with ASD}

Fig. 2 shows the results of the prediction analysis concerning SC impairments using machine learning models that are based on SVM algorithm. As shown in Fig. 2, the actual and predicted values of MBTP intervention-related outcomes of SC impairments almost coincided at some points. The machine learning model achieved a relatively high prediction effect given the fact that the model $(\mathrm{MSE}=0.188$, $\mathrm{RMSE}=0.434, \mathrm{R}^{2}=0.83$ ) predicted $83 \%$ of the variance in post-treatment SC impairments. 


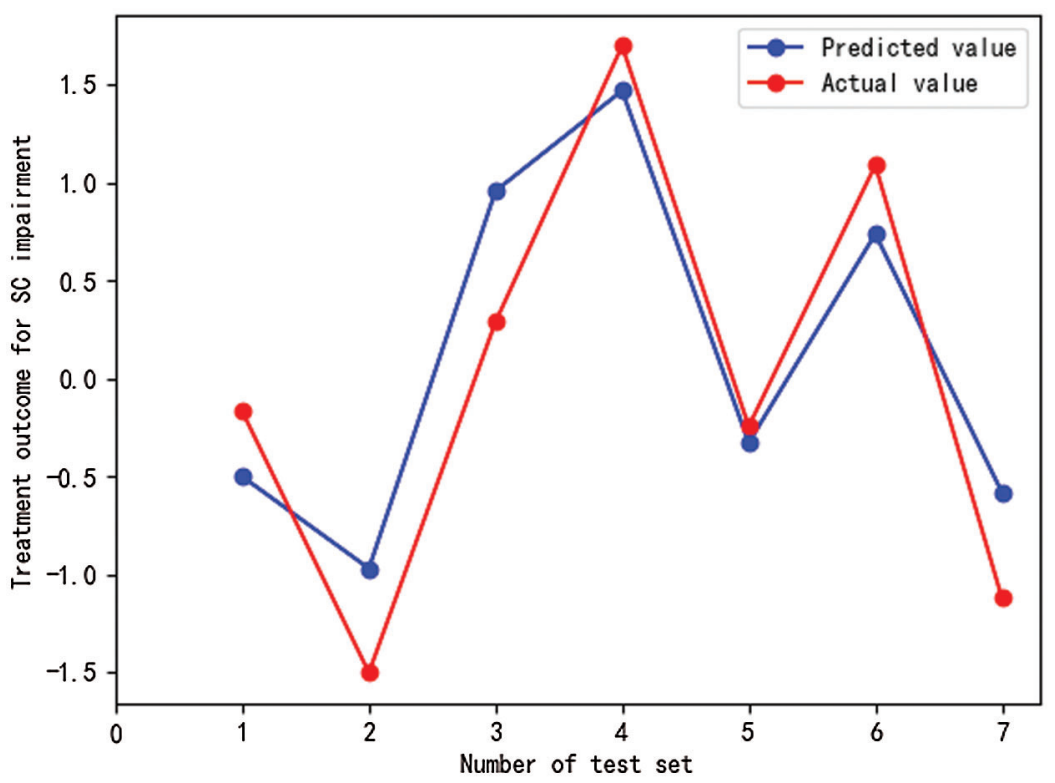

Figure 2: Prediction results of machine learning models for intervention-related outcomes of SC impairments in preschool children with ASD

Notes: SC impairments refers to social communication impairments, test sets are numbered from 1 to 7 . Drawn using the Matplotlib package in Python.

\subsubsection{Prediction Results of Machine Learning Models for Intervention-Related Outcomes of RRBs in} Preschool Children with ASD

Fig. 3 illustrate the results of the prediction analysis using a machine learning model that is based on SVM algorithm. As shown in Fig. 3, there were slight variations in actual values of RRBs when compared to predicted values. Our model $\left(\mathrm{MSE}=0.051, \mathrm{RMSE}=0.226, \mathrm{R}^{2}=0.859\right)$ predicted $85.9 \%$ of the variance in post-treatment RRBs.

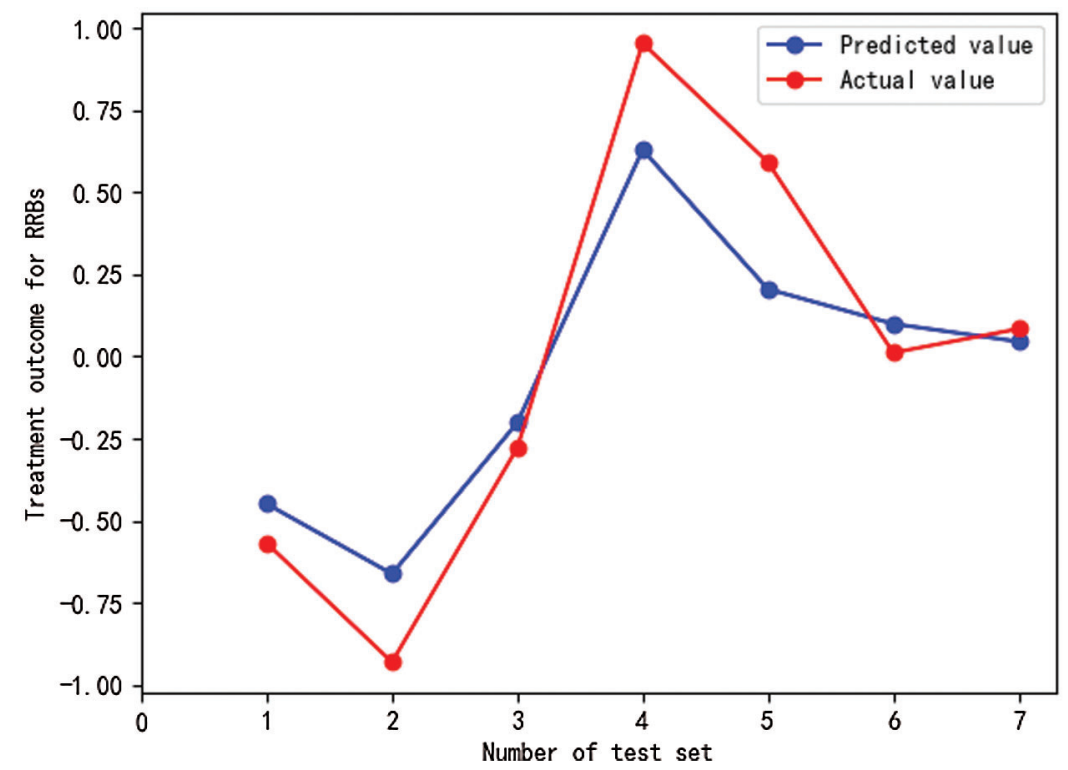

Figure 3: Prediction results of machine learning models for intervention-related outcomes of RRBs in preschool children with ASD

Notes: RRBs refers to restricted and repetitive behaviors, test sets are numbered from 1 to 7 . Drawn using the Matplotlib package in Python. 


\subsection{Comparisons of the Performance of Two Models}

We compared the performance of machine learning models and conventional statistical models in predicting the MBTP intervention-related outcomes of two core symptoms in preschool children with ASD (i.e., SC impairments and RBBs). In terms of predicting intervention-related outcomes of SC impairments in preschool children with ASD in response to MBTP, MSE and RMSE of the machine learning model were lower than those of the conventional statistical model, and the determination coefficient was increased by 0.234 . Hence, all three performance indicators of the machine learning model were better than those of the conventional statistical model (see Table 3). In terms of predicting intervention-related outcomes in RRBs in preschool children with ASD in response to MBTP, MSE and RMSE of the machine learning model were lower than those of the statistical model, while the determination coefficient was increased by 0.27 . The three performance indicators of the machine learning model were also superior to those of the statistical model (see Table 4).

Table 3: Comparison of the performance of two models in predicting intervention-related outcomes of MBTP on SC impairments in preschool children with ASD

\begin{tabular}{llll}
\hline Models & MSE & RMSE & $\mathrm{R}^{2}$ \\
\hline Statistical model & 0.455 & 0.675 & 0.596 \\
Machine learning model & 0.188 & 0.434 & 0.83 \\
\hline
\end{tabular}

Note: MSE is the mean square error, RMSE is the root mean square error, and $\mathrm{R}^{2}$ is the coefficient of determination. When MSE and RMSE are closer to 0 , the model's performance is better. When closer $\mathrm{R}^{2}$ is closer to 1 , the model's performance is better.

Table 4: Comparison of the performance of the two models in predicting intervention-related outcomes of MBTP on RRBs in preschool children with ASD

\begin{tabular}{llll}
\hline Models & MSE & RMSE & $\mathrm{R}^{2}$ \\
\hline Statistical model & 0.464 & 0.681 & 0.589 \\
Machine learning model & 0.051 & 0.226 & 0.859 \\
\hline
\end{tabular}

Note: MSE is the mean square error, RMSE is the root mean square error and $\mathrm{R}^{2}$ is the coefficient of determination. When MSE and RMSE are closer to 0 , the model's performance is better. When closer $\mathrm{R}^{2}$ is closer to 1 , the model's performance is better.

\section{Discussion}

\subsection{Predictors of Intervention-Related Outcomes for Two Core Symptoms in Preschool Children with ASD}

There is mounting evidence that physical interventions in general, and Mini-basketball training program (MBTP) in particular, can improve the core symptoms of ASD in preschool children (e.g., social communication (SC) impairments and restricted and repetitive behaviors (RBBs)). However, there is also growing evidence that not all preschool children benefit equally from such interventions as there is a considerable interindividual response variability in specific outcomes. However, currently there is a paucity of research which factors can predict such an interindividual response variability in response to physical interventions in preschool children with ASD. A better understanding of these predictive factors seems urgently needed to better personalize physical interventions which, in turn, is likely to maximize their effectiveness. Thus, this study investigate which factors in preschool children with ASD can predict MBTP intervention-related outcomes of SC impairment and RRBs. In this context, we observed that BMI is negatively correlated with intervention-related outcomes of RRBs. It has been previously reported that overweight and obesity rates are higher in preschool children with ASD than in healthy developing children [16]. Thus preschool ASD children with higher BMI might benefit to a lesser extent from MBTP intervention in terms of improvements of RRBs. The biological and psychological mechanisms driving 
this relationship are currently relatively unclear and require further investigations. Whether more personalized interventions (e.g., combining MBTP with nutritional intervention) being helpful to address this issue remains an interesting area for further investigations. We also observed that symptomatic severity is positively correlated with MBTP intervention-related outcomes of SC impairments and RRBs. This finding is consistent with previous reports suggesting that symptomatic severity considerably affects the core symptoms of preschool children with ASD [12,18]. Furthermore, this finding implies that MBTP had a greater effect on preschool ASD children with relatively severe symptoms which is perhaps driven by the fact there is larger room for improvements as compared to preschool ASD children with relative mild symptoms. Finally, there are positive correlation between baseline SC impairments, RRBS and their corresponding outcomes to the MBTP intervention. This finding is not surprising as it suggests that preschool children with higher SC impairments and RRBS at baseline benefit more than preschool children with already low baseline values.

\subsection{Prediction Results of the Statistical Models for Intervention-Related Outcomes of Two Core Symptoms in Preschool Children with ASD}

In this study, multiple linear regression models and machine learning based models were calculated and their performance was compared.

Multiple linear regression models were calculated to which extend in preschool children with ASD specific individual factors can predict the MBTP intervention outcomes on SC impairments and RRBs. The multiple linear regression models predicted $59.6 \%$ of the variance in post-treatment SC impairments and $58.9 \%$ of the variance in post-treatment RRBs. Although it is difficult to compare our findings to other studies given the fact that we are among the first applying such an approach to predict MBTP intervention-related outcomes in specific outcome parameters, the model performance in the current study differed from those of previous studies dealing with psychiatric disorders. Brousse et al. [43] predicted the treatment outcomes of antipsychotics on schizophrenia, however, the desired effect was not achieved, and the statistical model could only account for $28.1 \%$ of the variance in treatment outcomes. In another study [44], which predicted the outcomes of three drug treatments on depression, statistical models accounted for $25 \%, 43 \%$ and $71 \%$ of variances in treatment outcomes, respectively. Prediction performance of the statistical models were low, which might be because the traditional statistical models tend to rely on the existence of linear relationships, normal distribution and homogeneity of variance between data, which do not apply to the complex nature of data found in psychiatric research $[28,45,46]$.

\subsection{Prediction Results of Machine Learning Models for Intervention-Related Outcomes of Two Core Symptoms in Preschool Children with ASD}

We used machine learning models based on support vector machine algorithm to predict MBTP intervention-related outcomes of SC impairments and RRBs in preschool children with ASD. Machine learning models predicted $83 \%$ of the variance in post-treatment SC impairments and $85.9 \%$ of the variance in post-treatment RRBs, consistent with findings of studies on predicted treatment outcomes for children with obsessive compulsive disorders and depression. In the study of Lenhard et al. [46] comparable machine learning models was used to predict the treatment outcomes for Internet-delivered cognitive behaviour therapy in a cohort of children with obsessive compulsive disorder. Lenhard and colleagues found that their machine learning models could account for $75 \% \sim 83 \%$ of treatment outcomes in test set samples. Kim et al. [47] used machine learning models to predict the treatment outcomes of methylphenidate in ADHD children, and the accuracy of their machine learning model, which was based on support vector machine algorithm, reached $84.6 \%$. Collectively these findings suggest that machine learning models as emerging technology can yield to accurate and reliable predictions of interventionrelated outcomes in psychiatric disorders. 


\subsection{Comparisons of the Performance of Two Models in Predicting Intervention-Related Outcomes for Two Core Symptoms in Preschool Children with ASD}

The performance of the machine learning models was superior to statistical models in terms of MSE, RMSE and $\mathrm{R}^{2}$. The better performance of machine learning based algorithms is probably driven by the following facts. To begin with, The SVM algorithm in machine learning can map input data to a highdimensional feature space through nonlinear transformation to solved regression problems [48]. The Gaussian kernel function also could account for nonlinear relationships between variables. In contrast, statistical models require that data that need to satisfy some specific assumptions, otherwise resulting in poor model performance. Besides, machine learning models adopted the cross-validation method during training and obtained the best-performing model through multiple trainings, while the statistical models are not including such steps. Finally, machine learning and statistics had different focuses. The former focuses on data prediction, while the latter focuses on data interpretation and statistical inference [27]. In summary, machine learning is an effective way to predict treatment outcomes and can be used to guide decisions on preschool children with ASD that should be underwent a MBTP intervention. In this context, machine learning based algorithms might help to inform decisions making concerning personalized intervention options as they can account for important individual factors that can, to a certain extent, predict the intervention success.

\subsection{Limitations}

There were some limitations in this study that needs to be acknowledged. Firstly, the sample size was relatively small. However, previous studies achieved reliable results in prediction of intervention effects in obsessive-compulsive disorder [46] $(\mathrm{n}=61)$ and social anxiety disorder [49] $(\mathrm{n}=26)$ by comparable machine learning models. Furthermore, the SVM algorithm also has good fitting and generalization ability even for small samples sizes. Nevertheless, studies with large sample sizes are needed to substantiate our findings. Secondly, this study was based on data emerging from a single MBTP intervention with specific exercise and training variables. Thus, we are not able to rule out possible dose-response relationships between exercise and training variables with respect to changes of SC impairments and RRBs in preschool children with ASD. Such dose-response investigations are undoubtedly the basis for the development of more individualized intervention programs [50,51] (e.g., for preschool children with ASD). In future, high-quality studies with larger sample sizes are needed in children with ASD to investigate possible dose-response relationships regarding physical intervention characteristics and intervention outcomes of SC impairments and RRBs. Undoubtedly, data and knowledge obtained from such large-scale interventions would be helpful to further improve and better personalize the rehabilitation system for preschool children with ASD.

\section{Conclusions}

In summary, the findings of the current study suggest that symptomatic severity and baseline SC impairments are important factors that can predict, to a certain extent, the MBTP intervention-related outcomes of SC impairments in preschool children with ASD. In a comparable manner BMI, symptomatic severity, baseline SC impairments and baseline RRBs can predict, to a certain extent, the MBTP intervention-related outcomes of RRBs in preschool children with ASD. Machine learning models can successfully be applied to predict the MBTP intervention-related outcomes in preschool children with ASD, and peformed better than statistical models. Our findings inform on the selection of preschool children with ASD that are most likely to benefit from MBTP, and it provides a basis for the development of personalized intervention programs for preschool children with ASD. 
Author Contributions: Conceptualization, A.C. (Aiguo Chen); methodology, software, formal analysis, investigation, resources, visualization, and data curation, Z.S., K.C., X.D. and Z.L. (Zhimei Liu); writing - original draft preparation, Z.S. (Zhiyuan Sun); writing — review and editing, F.H., Q.Y., J.L. and L.Z. (Liye Zou). All authors have read and agreed to the published version of the manuscript.

Funding Statement: This research was supported by grants from the National Natural Science Foundation of China (31771243) and the Fok Ying Tong Education Foundation (141113) to Aiguo Chen.

Conflicts of Interest: The authors declare that they have no conflicts of interest to report regarding the present study.

\section{References}

1. Lord, C., Brugha, T. S., Charman, T., Frazier, T., Jones, E. J. H. et al. (2020). Autism spectrum disorder. Nature Reviews Disease Primers, 6(1), 694-701. DOI 10.1038/s41572-019-0138-4.

2. Bishop, S. L., Havdahl, K. A., Huerta, M., Lord, C. (2016). Subdimensions of social-communication impairment in autism spectrum disorder. Journal of Child Psychology and Psychiatry, 57(8), 909-916. DOI 10.1111/ jcpp. 12510.

3. Kanner, L. (1968). Autistic disturbances of affective contact. Acta Paedopsychiatrica, 35, 100-136. DOI 10.1111/ j.1651-2227.1968.tb06978.x.

4. Nadiga, A., Leeb, I., Singhb, L., Bossharta, K., Ozonoff, S. (2010). How does the topic of conversation affect verbal exchange and eye gaze? A comparison between typical development and high-functioning autism. Neuropsychologia, 48(9), 2730-2739. DOI 10.1016/j.neuropsychologia.2010.05.020.

5. South, M., Ozonoff, S., McMahon, W. M. (2005). Repetitive behavior profiles in Asperger syndrome and highfunctioning autism. Journal of Autism and Developmental Disorders, 35(2), 145-158. DOI 10.1007/s10803004-1992-8.

6. Maenner, M. J., Shaw, K. A., Baio, J., Washington, A., Patrick, M. et al. (2020). Prevalence of autism spectrum disorder among children aged 8 years - autism and developmental disabilities monitoring network, 11 sites, United States, 2016. MMWR Surveillance Summaries: Morbidity and Mortality Weekly Report, 69(4), 1-12. DOI 10.15585/mmwr.ss6904a1.

7. Liu, X., Lin, S., Chen, W., Yan, F., Shen, S. et al. (2018). Prevalence of autism spectrum disorders in Chinese children: A meta-analysis. Chinese Journal of Child Health Care, 26, 6. DOI 10.11852/zgetbjzz2018-26-04-15.

8. Bremer, E., Lloyd, M. (2016). School-based fundamental-motor-skill intervention for children with autism-like characteristics: an exploratory study. Adapted Physical Activity Quarterly, 33(1), 66-88. DOI 10.1123/ APAQ.2015-0009.

9. Ferreira, J. P., Ghiarone, T., Júnior, C. R. C., Furtado, G. E., Carvalho, H. M. et al. (2019). Effects of physical exercise on the stereotyped behavior of children with autism spectrum disorders. Medicina, 55(10), 685. DOI 10.3390/medicina55100685.

10. Wang, J., Cai, K., Liu, Z., Herold, F., Zou, L. et al. (2020). Effects of mini-basketball training program on executive functions and core symptoms among preschool children with autism spectrum disorders. Brain Sciences, 10(5), 263. DOI 10.3390/brainsci10050263.

11. Cai, K., Yu, Q., Herold, F., Liu, Z., Wang, J. G. et al. (2020). Mini-basketball training program improves social communication and white matter integrity in children with autism. Brain Sciences, 10(11), 803. DOI 10.3390/ brainsci10110803.

12. Dong, X., Chen, A., Liu, Z., Wang, J., Cai, K. (2020). Effects of mini-basketball training on repetitive behaviors and gray mattervolume in preschool children with autism spectrum disorder. China Sport Science and Technology, 56, 25-31. DOI 10.16470/j.csst.2020126.

13. Rosenblatt, L. E., Gorantla, S., Torres, J. A., Yarmush, R. S., Rao, S. et al. (2011). Relaxation response-based yoga improves functioning in young children with autism: A pilot study. Journal of Alternative and Complementary Medicine, 17(11), 1029-1035. DOI 10.1089/acm.2010.0834. 
14. Bishop, S. L., Richler, J., Lord, C. (2006). Association between restricted and repetitive behaviors and nonverbal IQ in children with autism spectrum disorders. Child Neuropsychology: A Journal on Normal and Abnormal Development in Childhood and Adolescence, 12(4-5), 247-267. DOI 10.1080/09297040600630288.

15. Hiller, R. M., Young, R. L., Weber, N. (2014). Sex differences in autism spectrum disorder based on DSM5 criteria: Evidence from clinician and teacher reporting. Journal of Abnormal Child Psychology, 42(8), 13811393. DOI 10.1007/s10802-014-9881-x.

16. Toscano, C. V. A., Ferreira, J. P., Gaspar, J. M., Carvalho, H. M. (2019). Growth and weight status of Brazilian children with autism spectrum disorders: A mixed longitudinal study. Jornal de Pediatria, 95(6), 705-712. DOI 10.1016/j.jped.2018.06.008.

17. Li, X., Liu, J., Yang, W., Cao, B. D., He, X. X. et al. (2012). Executive function, theory of mind, and symptom in children with high functioning autism. Chinese Mental Health Journal, 26, 594-589. DOI 10.3969/j.issn.10006729.2012.08.005.

18. Hodges, H., Fealko, C., Soares, N. (2020). Autism spectrum disorder: Definition, epidemiology, causes, and clinical evaluation. Translational Pediatrics, 9(S1), S55-S65. DOI 10.21037/tp.2019.09.09.

19. Tyler, K., MacDonald, M., Menear, K. (2014). Physical activity and physical fitness of school-aged children and youth with autism spectrum disorders. Autism Research and Treatment, 2014, 312163. DOI 10.1155/2014/312163.

20. Howard, J. (2019). Artificial intelligence: Implications for the future of work. American Journal of Industrial Medicine, 62(11), 917-926. DOI 10.1002/ajim.23037.

21. Rossi, A., Pappalardo, L., Cintia, P., Iaia, F. M., Fernàndez, J. et al. (2018). Effective injury forecasting in soccer with GPS training data and machine learning. PLoS One, 13(7), e0201264. DOI 10.1371/journal.pone.0201264.

22. Duarte, J. J., González, S. M., Cruz, J. C. (2020). Predicting stock price falls using news data: Evidence from the Brazilian market. Computational Economics, 57(1), 311-340. DOI 10.1007/s10614-020-10060-y.

23. Chekroud, A. M., Bondar, J., Delgadillo, J., Doherty, G., Wasil, A. et al. (2021). The promise of machine learning in predicting treatment outcomes in psychiatry. World Psychiatry, 20(2), 154-170. DOI 10.1002/wps.20882.

24. Jacobson, N. C., Nemesure, M. D. (2021). Using artificial intelligence to predict change in depression and anxiety symptoms in a digital intervention: Evidence from a transdiagnostic randomized controlled trial. Psychiatry Research, 295, 113618. DOI 10.1016/j.psychres.2020.113618.

25. Pearson, R., Pisner, D., Meyer, B., Shumake, J., Beevers, C. G. (2019). A machine learning ensemble to predict treatment outcomes following an Internet intervention for depression. Psychological Medicine, 49(14), 2330 2341. DOI 10.1017/S003329171800315X.

26. Askland, K. D., Garnaat, S., Sibrava, N. J., Boisseau, C. L., Strong, D. et al. (2015). Prediction of remission in obsessive compulsive disorder using a novel machine learning strategy. International Journal of Methods in Psychiatric Research, 24(2), 156-169. DOI 10.1002/mpr.1463.

27. Kagiyama, N., Shrestha, S., Farjo, P. D., Sengupta, P. P. (2019). Artificial intelligence: Practical primer for clinical research in cardiovascular disease. Journal of the American Heart Association, 8(17), e012788. DOI 10.1161/ JAHA.119.012788.

28. Breiman, L. (2001). Statistical modeling: The two cultures. Statistical Science, 16(3), 199-231. DOI 10.1214/ss/ 1009213725.

29. Schopler, E., Reichler, R. J., DeVellis, R. F., Daly, K. (1980). Toward objective classification of childhood autism: childhood autism rating scale (CARS). Journal of Autism and Developmental Disorders, 10(1), 91-103. DOI 10.1007/BF02408436.

30. Thorell, L. B., Nyberg, L. (2008). The childhood executive functioning inventory (CHEXI): A new rating instrument for parents and teachers. Developmental Neuropsychology, 33(4), 536-552. DOI 10.1080/ 87565640802101516.

31. Wei, W., Xie, Q., Zhu, J., He, W., Li, Y. (2018). The psychometric characteristics of childhood executive functioning inventory among chinese preschoolers. Chinese Journal of Clinical Psychology, 26, 26-29. DOI 10.16128/j.cnki.1005-3611.2018.01.006.

32. Bruni, T. P. (2014). Test review: Social responsiveness scale-second edition (SRS-2). Journal of Psychoeducational Assessment, 32(4), 365-369. DOI 10.1177/0734282913517525. 
33. Bodfish, J. W., Symons, F. J., Parker, D. E., Lewis, M. H. (2000). Varieties of repetitive behavior in autism: comparisons to mental retardation. Journal of Autism and Developmental Disorders, 30(3), 237-243. DOI 10.1023/A:1005596502855.

34. Martínez-González, A. E., Piqueras, J. A. (2018). Validation of the repetitive behavior scale-revised in spanishspeakers participants with autism spectrum disorder. Journal of Autism And Developmental Disorders, 48(1), 198-208. DOI 10.1007/s10803-017-3276-0.

35. Haga, A., Takahashi, W., Aoki, S., Nawa, K. (2019). Standardization of imaging features for radiomics analysis. The Journal of Medical Investigation, 66(1.2), 35-37. DOI 10.2152/jmi.66.35.

36. Thabtah, F., Kamalov, F., Hammoud, S., Shahamiri, S. R. (2020). Least loss: A simplified filter method for feature selection. Information Sciences, 534(4), 1-15. DOI 10.1016/j.ins.2020.05.017.

37. Scholkopf, B., Smola, A. J. (2000). New support vector algorithms. Neural Computation, 12(5), 1207-1245. DOI $10.1162 / 089976600300015565$.

38. Lee, I. G., Zhang, Q., Yoon, S., Won, D. (2020). A mixed integer linear programming support vector machine for cost-effective feature selection. Knowledge-Based Systems, 203(7), 106145. DOI 10.1016/j.knosys.2020.106145.

39. Yin, J. B., Li, T., Shen, H. B. (2011). Gaussian kernel optimization: Complex problem and a simple solution. Neurocomputing, 74(18), 3816-3822. DOI 10.1016/j.neucom.2011.07.017.

40. Lee, M. H., Ta, G. H., Weng, C. F., Leong, M. K. (2020). In silico prediction of intestinal permeability by hierarchical support vector regression. International Journal of Molecular Sciences, 21(10), 3582. DOI 10.3390/ijms21103582.

41. D’Este, M., Elia, M., Giannico, V., Spano, G., Lafortezza, R. (2021). Machine learning techniques for fine dead fuel load estimation using multi-source remote sensing data. Remote Sensing, 13(9), 1658. DOI 10.3390/ rs13091658.

42. Asif, A. A., Nishat, R., M., M., Faisal, F., Dip, R. R. et al. (2021). Performance evaluation and comparative analysis of different machine learning algorithms in predicting cardiovascular disease. Engineering Letters, 29, 731-741.

43. Brousse, G., Meary, A., Blanc, O., Lançon, C., Llorca, P. M. et al. (2010). Clinical predictors of response to olanzapine or risperidone during acute episode of schizophrenia. Psychiatry Research, 179(1), 12-18. DOI 10.1016/j.psychres.2009.03.003.

44. Kato, M., Serretti, A., Nonen, S., Takekita, Y., Wakeno, M. et al. (2015). Genetic variants in combination with early partial improvement as a clinical utility predictor of treatment outcome in major depressive disorder: The result of two pooled RCTs. Translational Psychiatry, 5(2), e513. DOI 10.1038/tp.2015.6.

45. Malley, J. D., Godlove Malley, K., Pajevic, S. (2013). Statistical learning for biomedical data. American Journal of Epidemiology, 177(3), 273-274. DOI 10.1093/aje/kws582.

46. Lenhard, F., Sauer, S., Andersson, E., Månsson, K. N., Mataix-Cols, D. et al. (2018). Prediction of outcome in internet-delivered cognitive behaviour therapy for paediatric obsessive-compulsive disorder: A machine learning approach. International Journal of Methods in Psychiatric Research, 27(1), e1576. DOI 10.1002/ mpr.1576.

47. Kim, J. W., Sharma, V., Ryan, N. D. (2015). Predicting methylphenidate response in ADHD using machine learning approaches. International Journal of Neuropsychopharmacology, 18(11), pyv052. DOI 10.1093/ijnp/ pyv052.

48. Jiang, F. (2020). Research on personal credit evaluation model of internet lending based on GA-SVM. Control Engineering of China, 27, 1025-1031. DOI 10.14107/j.cnki.kzgc.20180209.

49. Månsson, K., Frick, A., Boraxbekk, C. J., Marquand, A., Williams, S. et al. (2015). Predicting long-term outcome of Internet-delivered cognitive behavior therapy for social anxiety disorder using fMRI and support vector machine learning. Translational Psychiatry, 5(3), e530. DOI 10.1038/tp.2015.22.

50. Herold, F., Müller, P., Gronwald, T., Müller, N. G. (2019). Dose-response matters!-A perspective on the exercise prescription in exercise-cognition research. Frontiers in Psychology, 10, 2338. DOI 10.3389/fpsyg.2019.02338.

51. Herold, F., Törpel, A., Hamacher, D., Budde, H., Gronwald, T. (2020). A discussion on different approaches for prescribing physical interventions-four roads lead to rome, but which one should we choose? Journal of Personalized Medicine, 10(3), 55. DOI 10.3390/jpm10030055. 


\section{Appendix A}

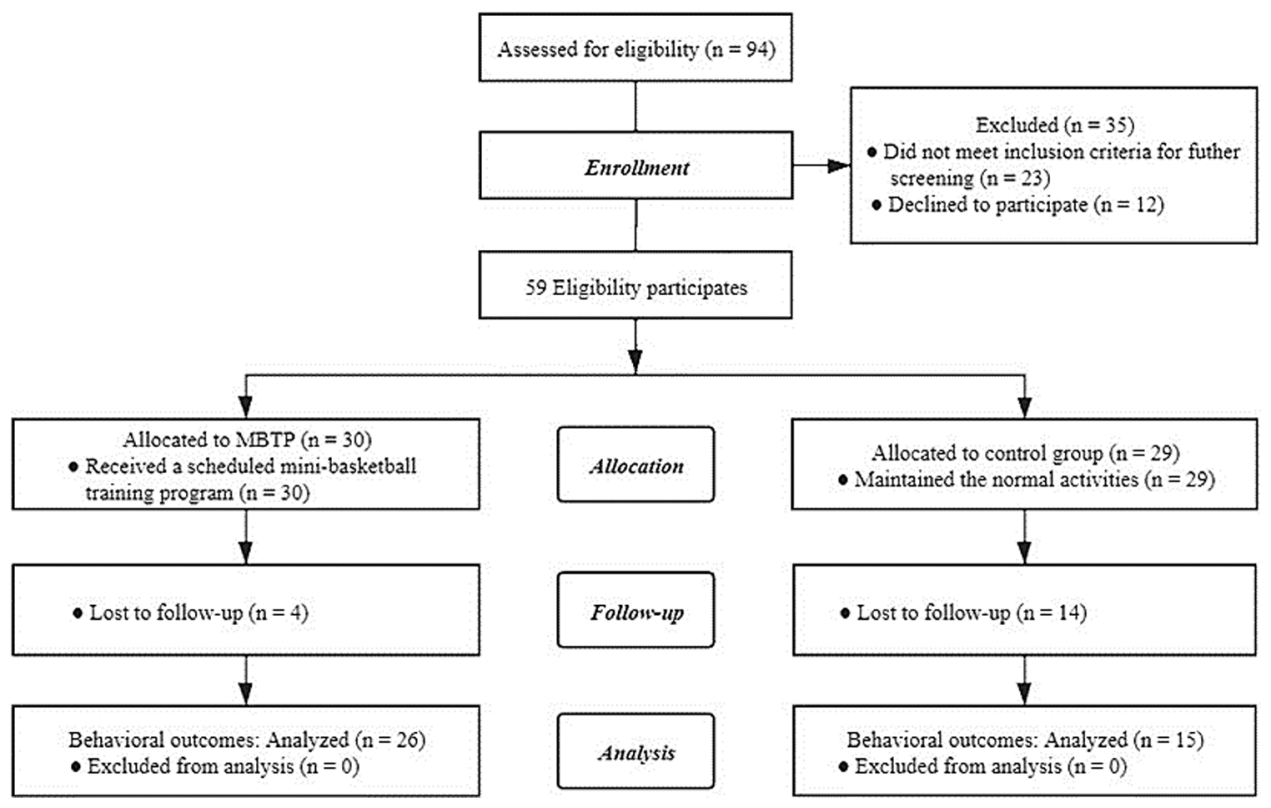

Figure A1: Participant flow chart 